\title{
BIM y Madera. Nuevos desafíos para el Diseño y Construcción
}

\author{
BIM and Wood. New challenges for Design and Construction
}

\author{
Danny Lobos \\ UDLA, Chile \\ dlobos@udla.cl \\ Clara Codron Lechuga \\ Pontificia Universidad Católica de Chile, Chile \\ cacodron@uc.cl
}

\author{
Felipe Pino de la Cruz \\ Pontificia Universidad Católica de Chile, Chile \\ fipino@uc.cl \\ Victor Nunez Bustos \\ Pontificia Universidad Católica de Chile, Chile \\ vgnunez@uc.cl
}

\begin{abstract}
The work compiles several academic and Research initiatives, and aims to establish a right link between two agendas that governments, AEC industry and academia normally handle separated, this is BIM (Building Information Modeling) and Wood. By running several literature reviews, interviews and software tests, the state-of-the-art was reached in both fields; several cases linking BIM and wood are shown and discussed. It can be concluded that both fields have several commons processes and also that many cases have used just a few BIM tools, disregarding a big potential of these methodologies.
\end{abstract}

Keywords: Wood; BIM (Building Information Modeling); Architectural Design; Building Construction.

\section{Introducción}

El presente trabajo recopila una serie de iniciativas académicas y de investigación, tendientes a establecer la correcta vinculación entre dos agendas de gobierno, industria y academia que, en general, se manejan por separado, hablamos de BIM (Building Information Modeling) y la Madera. Mediante revisiones bibliográficas, entrevistas y testeo de software se recopila el estado del arte en ambas disciplinas, se muestran y discuten diversos casos donde estas agendas han actuado en conjunto. Se concluye que ambos procesos tienen importantes puntos de convergencia y algunos procesos pendientes, y que los actuales casos de estudio de BIM para el diseño y la construcción en madera solo han usado aspectos puntuales de las metodologías BIM.

\section{Situación BIM en el mundo y en Chile}

Las definiciones más aceptadas por la academia respecto a los conceptos y software que fundamentan BIM, corresponden, a las de ISO Standard (ISO, 2010) la cual habla de una "representación digital compartida de las características físicas y funcionales de un objeto construido, con el objetivo de ser una base confiable para tomar decisiones"

En términos más prácticos las herramientas BIM son usadas para generar modelos 3D y 4D, la visualización, generación automática de dibujos y tablas de cuantificación, detección de interferencias (Imagen 1). En general son útiles en todas las etapas de proyecto (anteproyecto-> proyecto->construcción>operación->renovación) (Eastman, 2011; Kryegel y Nies, 2008).

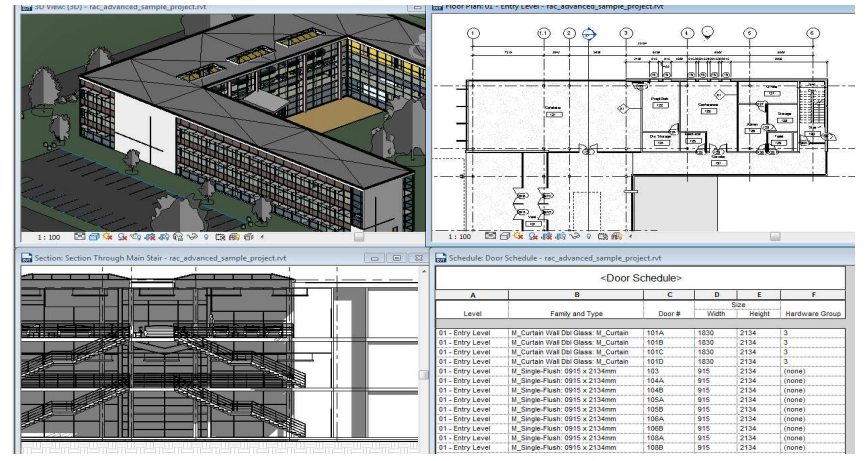

Figura 1: Un edificio modelado en BIM Revit. Se muestra vista 3d, planta, corte y cubicación de puertas. Fuente: Elaboración propia, 2015

Algunos ejemplos de software BIM (Imagen 1) son Revit, Archicad, Tekla, Allplan, AECOSim (Khemlani, 2010). Sin embargo la implementación BIM no es solo software sino que incluye procesos y supervisión (CICRG, 2010). De los anteriores solo Archicad había creado desde sus inicios herramientas para madera (ArchiFrame y Trussmaker).

Existe una interfaz de programación (API, Advanced Programming Interface) para crear herramientas nuevas en cada BIM (Kryegel, 2008; Soto and Moa, 2013; Lobos et all, 2014; Dzambazova et all, 2008).

Los cursos BIM se dictan desde hace 15 años en diversas universidades chilenas (principalmente universidades UDLA, USACH, UChile, PUC, DUOC, UDP, UBioBio, etc) y existe una amplia masa de usuarios que está trabajando en el mercado (Loyola, 2016).

Es obligatorio usar BIM para edificación pública en Estados Unidos (GSA, 3D-4D Program), Inglaterra (Government 
Construction Strategy) y Singapur. En Chile es obligatorio en hospitales y existen algunos proyectos del MOP (Ministerio de Obras Públicas) en curso que lo exigen: Centro Cultural Gabriela Mistral, ampliación Américo Vespucio Oriente, Teatro Regional del BioBio, Puente Canal de Chacao. La empresa BIM-Chile fue una de las primeras en apoyar la implementación de BIM en gobierno desde 2012, posteriormente, desde 2016 se iniciaron paralelamente Plan BIM de CORFO y BIM Forum de la CDT.
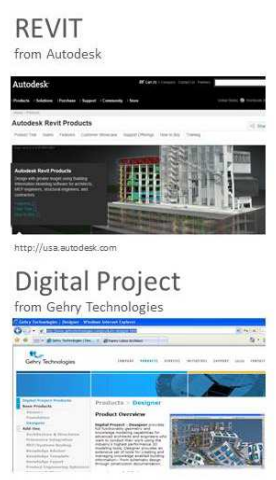

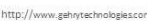
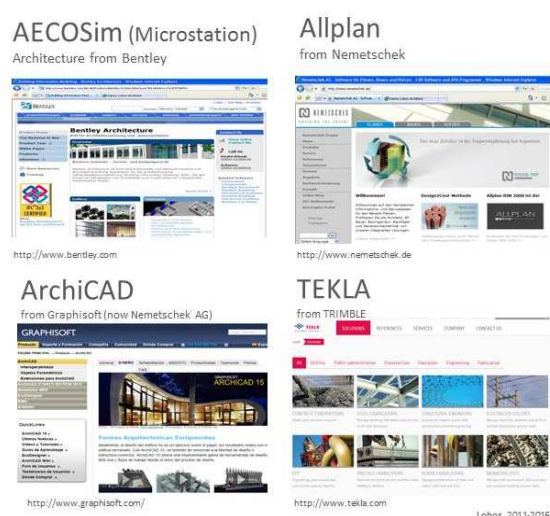

TEKLA

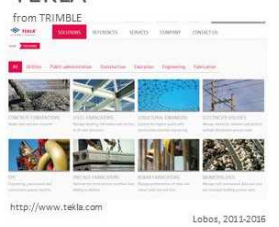

Figura 2: Los software BIM más comunes en el mercado. Fuente: Elaboración Propia, 2015

\section{Situación de la madera en el mundo}

Hace más de 20.000 años que existe la construcción en madera en el mundo. Pero alrededor de los años 1850, con la aparición del hormigón, la construcción en madera fue desapareciendo. Sin embargo, desde los años 60, con los problemas medioambientales, la demanda para volver a la construcción en madera ha aumentado. (CSTB, 2006)

En los países del Norte de Europa como Suecia, Finlandia y Noruega, (regiones altamente concentradas en madera blanda o resinosos), desde hace mucho tiempo, se construye en madera sobre todo por las condiciones del clima y por los recursos disponibles en estas zonas. La combinación estos dos factores hacen que se construya por más de $95 \%$ en viviendas particulares.

En los países con alta cobertura forestal, como Canadá (347 millones de ha) y Estados Unidos (310 millones de ha), la construcción en madera se ha desarrollado rápidamente con las aportaciones de técnicas nuevas, hasta alcanzar hoy en día alrededor de $90 \%$ de construcciones en madera. (CSTB, 2006)

Se ha demostrado mediante la exhaustiva y documentada experiencia de los países del Hemisferio Norte, que utilizan la madera como sistema constructivo válido, que ésta es el material ideal para la construcción por sus cualidades físicas y mecánicas y por sus ventajas medioambientales.

1 El 23\% corresponde a especies de Eucalipto, el resto a otras especies.
La madera aporta muchos beneficios como: su buen desempeño antisísmico, su resistencia al fuego, su calidez y bienestar para el usuario, su bajo costo, y su rapidez de ejecución (Madera 21, 2017).

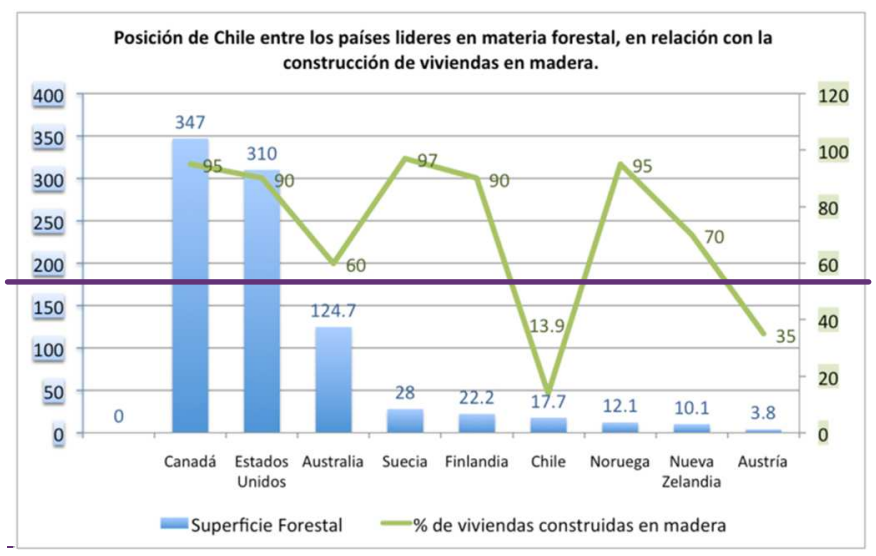

Figura 3: Posición de Chile entre los países líderes en materia forestal, en relación con la construcción de viviendas en madera.

Fuente: Elaboración Propia, 2017 (desde CONAF, FAO y CSTB)

Entrega también una respuesta favorable a la preocupación del medioambiente. Es un material renovable, su transformación necesita poca energía y permite reducir las emisiones de $\mathrm{CO} 2$, principal causante del cambio climático. (Madera 21, 2017). Efectivamente en cada metro cúbico de un producto en madera, quedan atrapadas alrededor de unas 0.9 t de $\mathrm{CO} 2$, a lo largo del ciclo de vida de un producto (CEIBois, 2009).

\section{Madera en Chile}

La superficie cubierta de bosques a nivel nacional representa el $23 \%$ del total (CONAF, 2017) es decir 17,7 millones de hectáreas en 2015 (FAO, 2015), de las cuales, aproximadamente el $80 \%$ corresponde a los bosques nativos (13,6 millones de há), y el 20\% a plantaciones forestales $(2,87$ millones de há), posicionando a Chile como uno líder en materia forestal (Imagen 3) y a sus bosques nativos como unos de los ecosistemas más escasos y valorados del mundo" (CONAF, 2013).

Específicamente, el Pino Radiata corresponde al 68\% de las plantaciones forestales ${ }^{1}$, (CONAF, 2017), siendo Chile uno de los diez mayores productores de madera del mundo (CIM, 2015) y líder mundial, junto con Nueva Zelanda, en cuanto a Pino Radiata (Madera 21, 2017).

Además, el sector forestal es el segundo exportador del país, con un 3,1\% de participación en el PIB (2010), actividad basada principalmente en la celulosa, pero también en tableros y madera aserrada, estos últimos, principales productos de la construcción en madera, enviados a más de 100 países en los 5 continentes (CONAF, 2013). 
Chile tiene entonces, los recursos necesarios para desarrollar la construcción en madera y convertirla en un sistema constructivo válido, pudiendo las plantaciones forestales chilenas abastecer la industria por un tiempo indefinido, generando beneficios económicos, sociales y ambientales (Madera21, 2017).

Sin embargo, se construye muy poco en madera en el país (14\% de las viviendas en el país), existiendo al menos cinco factores que impiden su desarrollo (Madera21, 2017):

- Percepción poca valorizada que tiene el cliente final con respecto a las viviendas en madera.

- Desconocimiento y prejuicios de la gente frente a este material.

- $\quad$ Normativas inadecuadas que regulan el uso de la madera imponiendo restricciones.

- Baja tasa de certificación que incita a la mayoría de las empresas a optar por construir con otros materiales.

- $\quad$ Falta de especialistas y profesionales capacitados para construir viviendas de calidad en madera.

Por otro lado, en 2014 nace en Chile el Programa Construye 2025, cuyo objetivo es transformar el sector de la construcción desde la perspectiva de la sustentabilidad y productividad al año 2025, incluyendo las líneas de BIM y madera, por separado.

\section{Diseño y construcción en madera}

En su etapa de diseño, la construcción en madera se caracteriza como: un "diseño por protección" es decir, con varias capas que protegen los distintos elementos incorporados dentro de ellas; pero también un "diseño por ensamblajes", donde las piezas están unidas mediante una diversidad de soluciones de fijaciones que juntas determinan la estabilidad de la estructura. Esto último se logra a través de una lógica caracterizada por parámetros dimensionales y de ubicación (dimensiones, distanciamiento, ángulo, etc.).

Además, la construcción en madera requiere prefabricación, con el fin de disminuir su tiempo de ejecución, debiendo el diseño representar con alta precisión sus elementos. La estabilidad de la estructura y la protección del edificio dependen de la calidad de la documentación elaborada en la etapa de diseño, cuyos errores $u$ omisiones se reflejan en la etapa de construcción y afectan la calidad de la obra.

Finalmente, durante su etapa de uso, la madera requerirá mantenimiento debido a sus propiedades biológicas, que la hacen vulnerable frente a exigencias medio ambientales.

\section{Tecnologías CAD que apoyan el diseño en madera}

En la actualidad, la mayoría de los profesionales utilizan como método de elaboración de proyecto el método tradicional con base CAD (Computer-Aided-Design), presenta varias dificultades (CDT 2010), como: tiempo elevado de elaboración en la etapa de diseño y errores que afectan su eficiencia y tienen repercusiones en la etapa de construcción y en la calidad de la obra final. Dada la especificidad y complejidad del diseño en madera, es necesario el uso de herramientas de trabajo que cautelen su calidad a lo largo de todo su ciclo de vida.

La calidad de la obra final de una construcción en madera dependerá, en gran parte, de las herramientas de trabajo en las etapas de diseño, de construcción y de operación de un proyecto, teniendo las nuevas tecnologías BIM el potencial de mejorar este aspecto.

\section{Ecosistema BIM-madera}

Es necesario gestionar toda la información que proviene de un edificio con estructura en madera, así como tener una buena manipulación de los parámetros y reglas de ensamblaje para lograr un diseño eficiente.

Las herramientas BIM son adecuadas para el diseño en madera porque permiten incorporar y gestionar toda la información relevante de un proyecto en un modelo $3 D$ y además poseen un grado de "inteligencia" por su diseño paramétrico. "Los elementos (muros, vigas, ventanas, puertas, etc.), antes representados por propiedades fijas (largo, ancho y alto), son caracterizados por parámetros y reglas que determinan la geometría del edificio." (Rojas, 2011).

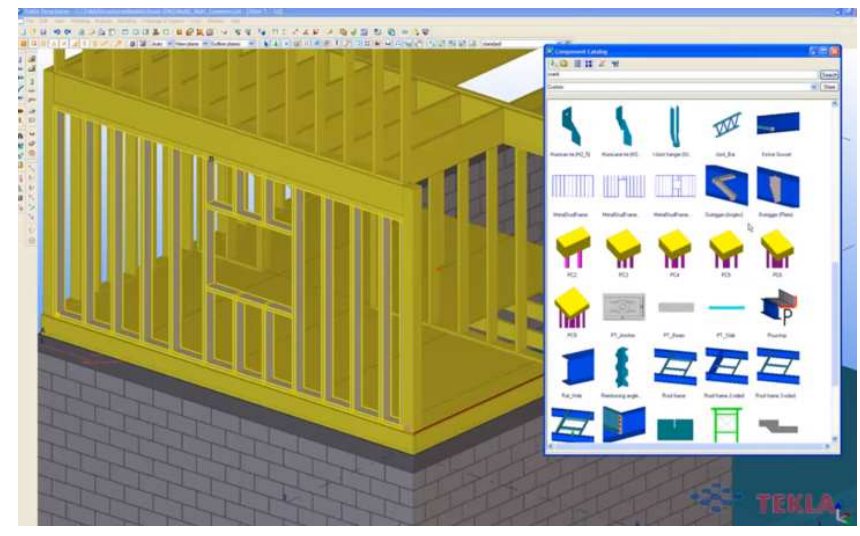

Figura 4: Modelo TEKLA para Madera. Fuente TEKLA USA Webinars, 2010

Actualmente, existen en el mercado algunas aplicaciones adicionales para BIM (plugins) especializadas en la construcción en madera, cuyo objetivo es mejorar la eficiencia del diseño, como ArchiFrame, AGACAD Wood Framing o WoodStud_Frame (ver Tabla 1), así como extensiones de los software nativos BIM Archicad (Graphisoft), Revit (Autodesk) y Tekla, respectivamente.

Estos, permiten extender las funcionalidades de los softwares BIM nativos a herramientas más completas para el diseño y concepción de edificios en madera mediante comandos y sequencias para el diseño de entramados verticales, columnas, vigas, entramados horizontales, y techumbres. Al contrario de los softwares 2D, que se caracterizaban por ser del tipo manual, con dichas aplicaciones BIM, no se requiere mayor elaboración en el diseño del paquete constructivo, ya que configura y diseña de manera automática. 
Específicamente por ejemplo, Archiframe permite calcular materiales, diseñar para CNC, crear los entramados y vigas en 3D y elevaciones en 2D, mientras que WoodStud_Frame funciona con bloques listos de paneles paramétricos en madera incluyendo conectores metálicos y pernos (aunque estos últimos son bloques fijos no paramétricos en su mayoría).

Tabla 1 Nombre, Fabricante y Costos de software BIM-Madera. Fuente Elaboración propia, 2017.

\begin{tabular}{|lll|}
\hline Nombre & Fabricante & Costo Aprox \\
\hline ArchiFrame & ArchiFrame & 500Euros \\
\hline AGACAD Wood Framing & AGACAD & 400USD \\
\hline WoodStud_Frame & Tekla & -- \\
\hline Metal Wood Framer & $\begin{array}{l}\text { StrucSoft } \\
\text { Solutions Ltd. }\end{array}$ & -- \\
\hline Timber Framing 2015 & Autodesk & $\begin{array}{l}\text { Clientes con } \\
\text { suscripción }\end{array}$ \\
\hline
\end{tabular}

\section{Funciones transversales de las herramientas BIM para madera}

Las extensiones mencionadas poseen funciones muy parecidas que se benefician de las herramientas básicas de diseño de los software nativos BIM (todas las vistas se actualizan automáticamente después de haber diseñado o modificado un elemento en el diseño, a diferencia con los software 2D, donde es necesario generar y actualizar cada vista manualmente).

Además, toda la información del modelo queda guardada en una base de datos para cada etapa del ciclo de vida del proyecto, como construcción, mantenimiento y uso (Eastman et all, 2011).

\section{Funciones específicas para BIM en madera}

Extensiones como Agacad, 2017 y Archiframe 2017, llamados en adelante software BIM-Madera, poseen herramientas específicas para el diseño de una construcción en madera, como:

- Un sistema multicapas: donde el diseño de una construcción en madera se caracteriza por tener un sistema de varias capas (revestimiento interior, componente estructural, revestimiento exterior, etc.), permite configurar, crear y generar con alta precisión cada una de las capas con una cierta flexibilidad y controlar cada uno de sus elementos, así como el conjunto.

- Configuración y generación automatizada de entramados: donde las herramientas de diseño permiten definir sus componentes (pie derechos, paneles, revestimientos, vigas, cadeneta, etc.) configurando los parámetros dimensionales (escuadría, espesor, alturas etc.) y de ubicación (distanciamiento, ángulo, etc.) para luego generarlos de manera automática. En caso de cambio en el diseño arquitectónico y/o estructural, los entramados y sus componentes se actualizan automáticamente. También tiene la posibilidad de configurar las conexiones entre pie derechos en $\mathrm{L}, \mathrm{T} u$ otras conexiones, lo que permite modelar con flexibilidad y manejar fácilmente situaciones complejas. En un gran proyecto, de varios edificios en madera, es posible diseñar múltiples entramados de manera automatizada.

- Configuración y generación de la documentación: donde se generan automáticamente láminas con distintas vistas que presentan una o varias capas de un componente (entramado vertical, entramado horizontal, entramado de techumbre) según lo predefinido en el patrón. El proceso de documentación se vuelve automatizado y rápido, ideal para la larga elaboración de planos de montaje y fabricación, que por lo general es larga y tediosa.

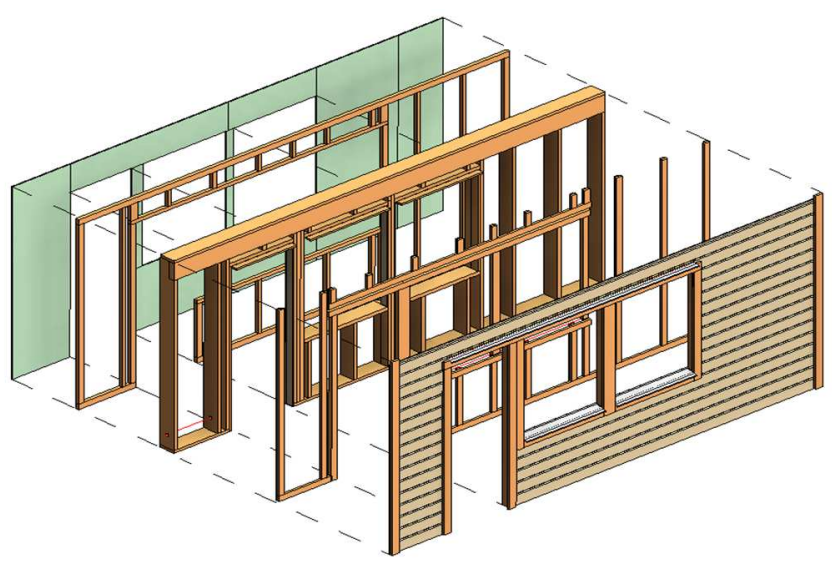

Figura 5: Modelo AGACAD Wood Framing, Sistema Multi Capas. Fuente AGACAD Wood Framing, 2017.

Otras herramientas: donde por ejemplo es posible diseñar en madera maciza; la posibilidad de exportar un modelo de entramado con varias capas desde los softwares hacia las líneas de producción de paneles automatizados a través de máquinas CNC. De esta manera es posible predefinir directamente diferentes operaciones para cada etapa de la línea de producción, incluyendo aserrado, perforación, corte y marcado para cada elemento.

\section{Conclusiones sobre herramientas BIM-Madera}

Todas estas herramientas logran mejorar la eficiencia del diseño de una construcción en madera, disminuyendo el tiempo pasado en el diseño, mejorando la comprensión y visualización del proyecto de manera a ayudar la toma de decisiones e intercambiando información de manera eficiente. Entonces se agiliza el flujo de trabajo, aumenta la productividad y mejora la calidad de la obra final.

Sin embargo, existen frenos al uso de estas herramientas. Efectivamente, uno de los frenos destacados es el tiempo importante dedicado a la configuración de los distintos parámetros del software con respecto al proyecto. Sin una correcta configuración, es muy difícil obtener un diseño eficiente. También las extensiones BIM pueden parecer poco flexibles por su carácter paramétrico y automatizado al contrario de las herramientas de los software CAD, que son conocidas por entregar una cierta libertad en el diseño. 
Es necesario conocer muy bien los softwares y sus herramientas BIM para lograr una mayor eficiencia. Además, deben considerarse los costos de licencias BIM, más los plugins, las capacitaciones, interoperabilidad de los formatos con la industria local.

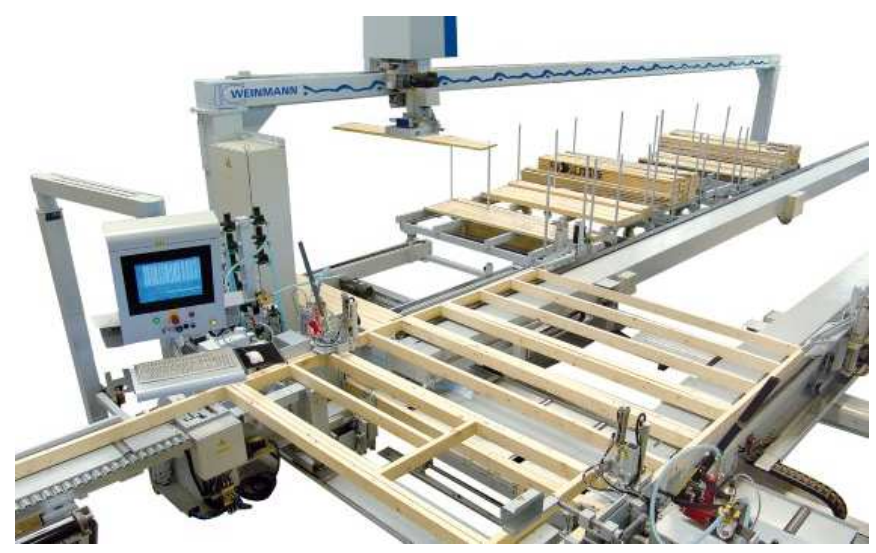

Figura 6: Sistema de cortes CNC. Fuente: 4.bp.blogspot.com

\section{EJEMPLOS}

A continuación, un breve análisis de casos documentados en el mundo donde se destaca la aplicación de BIM para resolver temas de diseño y construcción en madera.

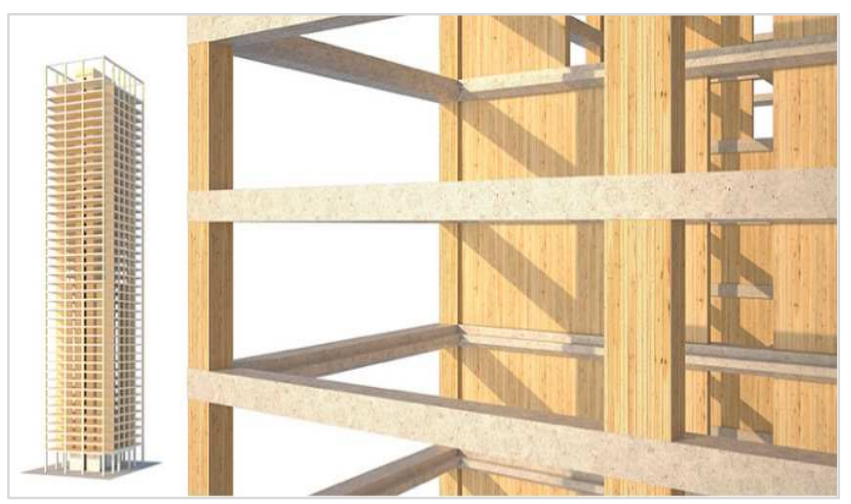

Figura 7: SOM's proposal for a 40-story using BIM and CLT in High Rise Buildings. Source: archpaper.com

\section{SOM}

Es una propuesta (no construida) para el uso de BIM en edificios de gran altura (Imagen 7), se trata de una torre de madera de 40 pisos con un núcleo de concreto basado en un sistema estructural diseñado por Fazlur Kahn en 1965.

\section{Empresa Metsawood}

Metsawood ofrece Ingeniería de madera a través de BIM, permite la coordinación entre las disciplinas de diseño y además la comunicación del diseño a la obra, bajo el enfoque de ingeniería digital BIM apoya desde la fase de planificación y diseño del proyecto, extendiéndose a lo largo del ciclo de vida del edificio, soportando procesos que incluyen la gestión de costes, la gestión de la construcción, la gestión de proyectos y el funcionamiento de las instalaciones.

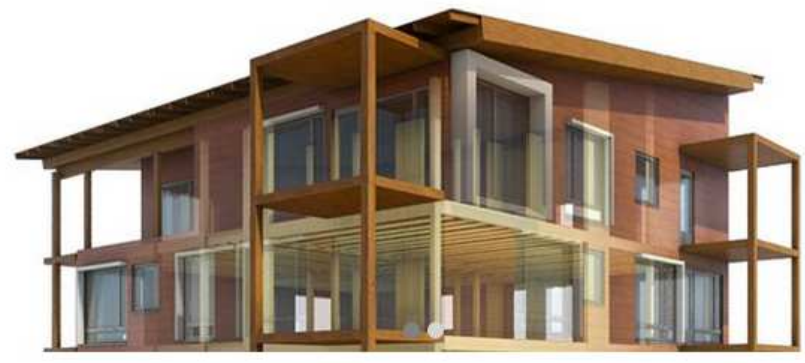

Figura 8: Modelo BIM de vivienda en madera. Fuente: Empresa Metsawood

\section{Ejemplos académicos en Chile}

A continuación algunos ejemplos académicos de integración BIM y madera realizados por arquitectos y constructores al alero del programa de Magister de la Universidad Católica y la Universidad de las Américas.

El siguiente ejercicio académico del prof. Dr-Ing Danny Lobos parte de la hipótesis de que se puede incorporar análisis estructural en base al método AM (Analitical Models) para el análisis y simulación de la estructura de un diseño propuesto de 12 pisos (casa modulo visto equivale a dos pisos)

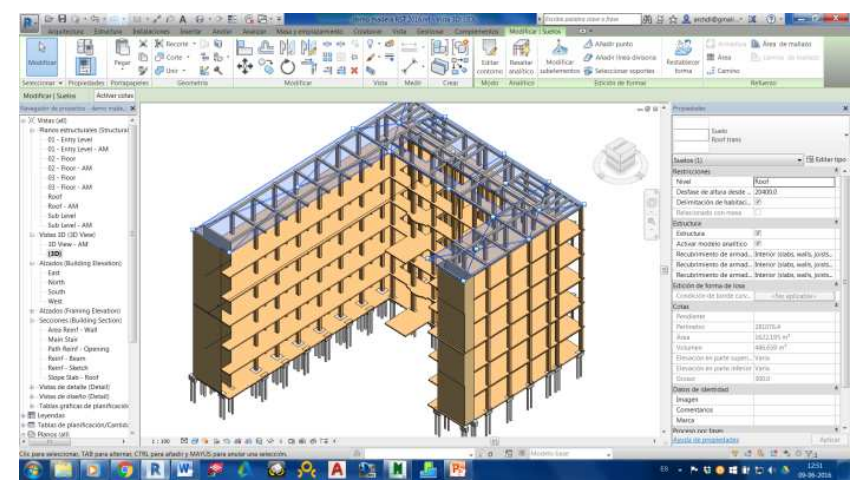

Figura 9: Modelo estructural de edificio de 12 pisos en madera hecho en Autodesk Revit. Fuente: Prof Danny Lobos, 2016

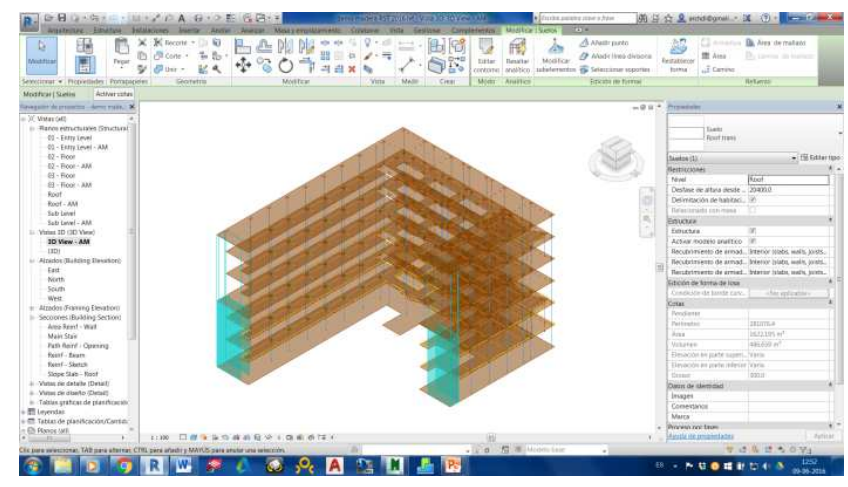

Figura 10: Modelo analítico estructural de edificio de 12 pisos en madera hecho en Autodesk Revit. Fuente: Prof Danny Lobos, 2016 


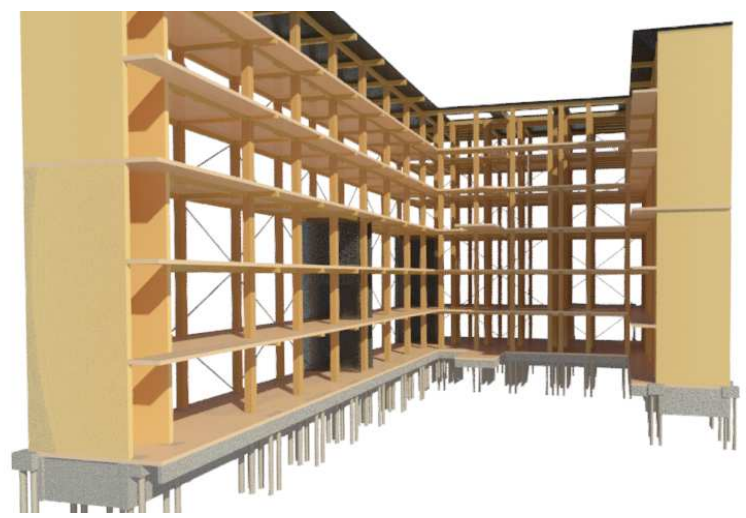

Figura 11: Vista perspectivada del modelo estructural de edificio de 12 pisos en madera hecho en Autodesk Revit. Fuente Prof Danny Lobos, 2016

El Constructor Civil y Magister PUC @ Oscar Merino realizó un estudio exploratorio donde revisó innovaciones en la edificación en madera los países desarrollados (Europa, Norteamérica y Oceanía), determinando el estado de la tecnología con respecto a este material, y su aplicación en viviendas de hasta 3 pisos. Por otro lado ha logrado indagar a la fecha sobre el potencial de las metodologías BIM (sus procesos y etapas), con el fin de contrastar las distintas etapas de una edificación con madera (de última generación tanto con métodos tradicionales) con los métodos BIM.

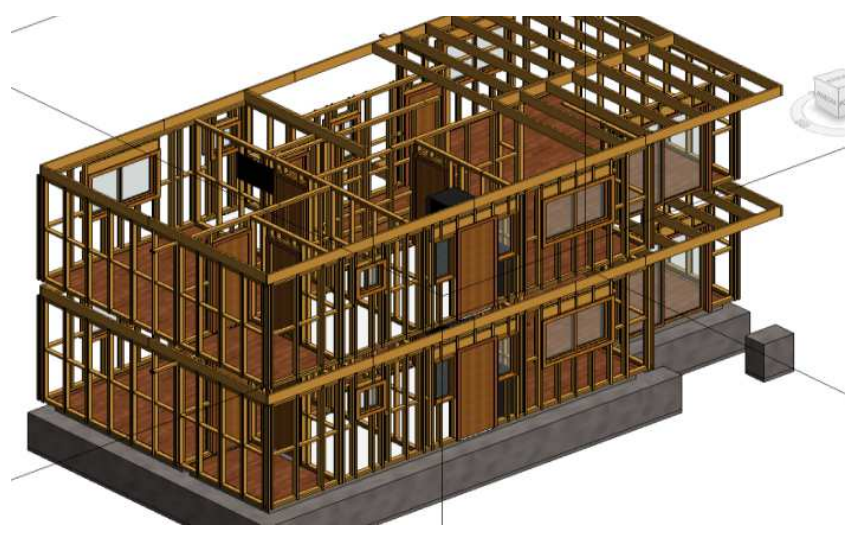

Figura 12: Vista isométrica del diseño vivienda 2 pisos en madera modelada con software BIM. Fuente: Oscar Merino, Tesis UC, 2014

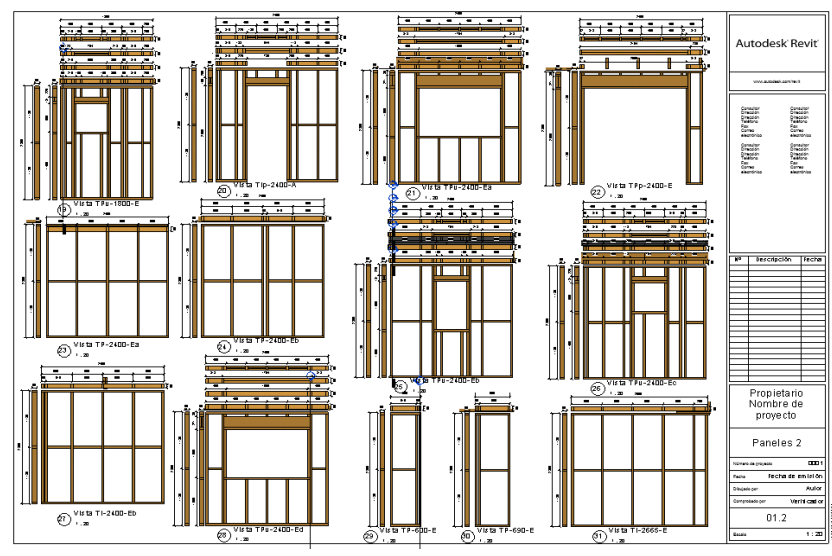

Figura 13: Planos de detalle de vivienda 2 pisos en madera modelada con software BIM. Fuente: Oscar Merino, Tesis UC, 2014
El Constructor Civil Felipe Pino realizó dos ejercicios de exploración de la aplicación de la herramienta AGACAD en la resolución concreta de un diseño de dos pisos en madera, donde pudo evidenciar ventajas en la visualización, modelo 3d (en base a familias y assemblies), planos, cubicaciones. Sin embargo la creación de las plantillas nacionales toma bastante tiempo y además la resolución algunos encuentros especiales no está parametrizada.

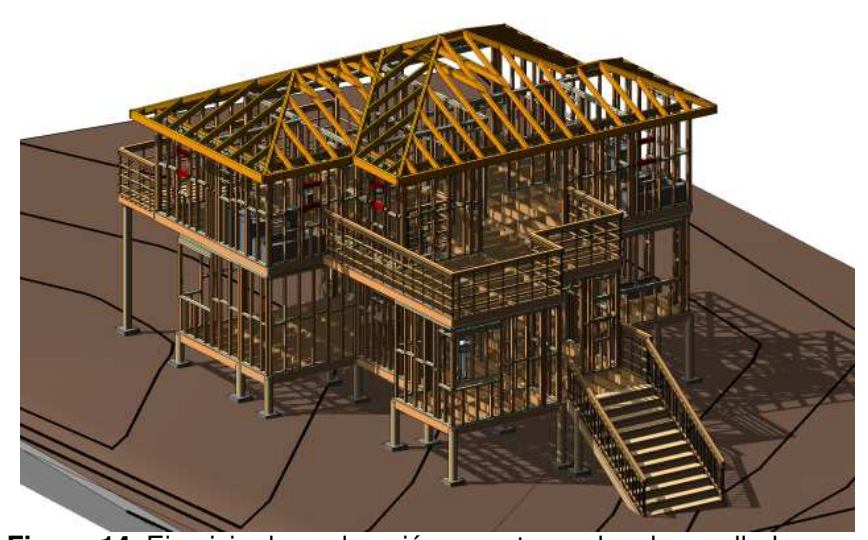

Figura 14: Ejercicio de exploración en entramados desarrollados con herramienta BIM AGACAD. Fuente: Felipe Pino, 2016

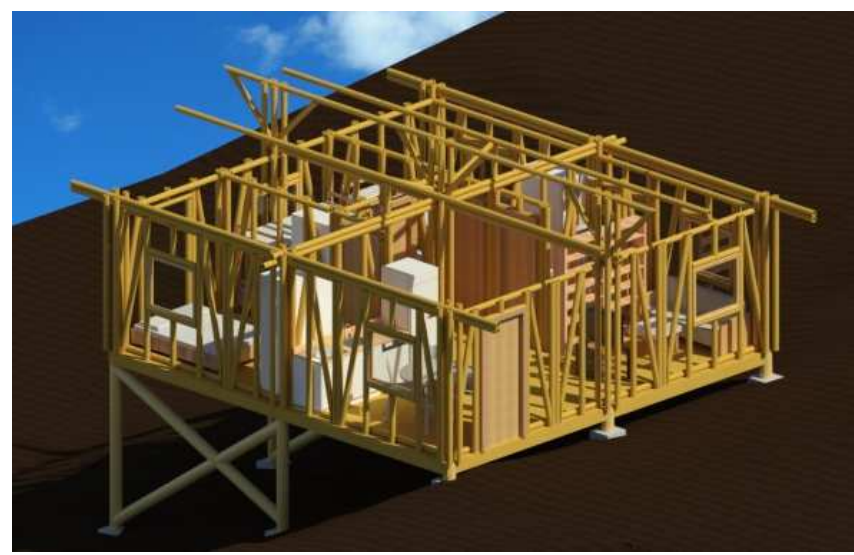

Figura 15: Ejercicio de exploración de herramienta BIM AGACAD para diseño en bambú. Fuente: Felipe Pino, 2016

El arquitecto Victor Nuñez exploró la interoperabilidad facilitada por el BIM. Privilegiando el dialogo del diseñador con el mandante, con los especialistas y al momento de construir. Realizó una serie de tests con diversos plugins para Archicad (BIM de Graphisoft) donde constató rebajas sustanciales (casi a un tercio) del tiempo de los procesos, e incluso, logrando hacer muchas más correcciones dentro del tiempo limitado en el proyecto específico. Archiframe es una completa herramienta de diseño para estructuras de madera, proporciona herramientas para el diseño de estructuras de pared, columnas y vigas, pisos intermedios y estructuras de tejado. Estas herramientas hacen posible remodelar tablones y vigas de cualquier manera para obtener el resultado deseado. Todo dentro de un solo archivo - incluyendo modelos 3D y elevaciones 2D con diseños semiautomáticos y listados. Las piezas de madera individual (tablones) se pueden fabricar manualmente usando dibujos de la dimensión y listas de corte, o se producen con CNC. Además de conectar todo el modelo 
integrado a MEP (Mechanical Electrical and Plumbing, o sea clima, electricidad y agua potable/alcantarillado).

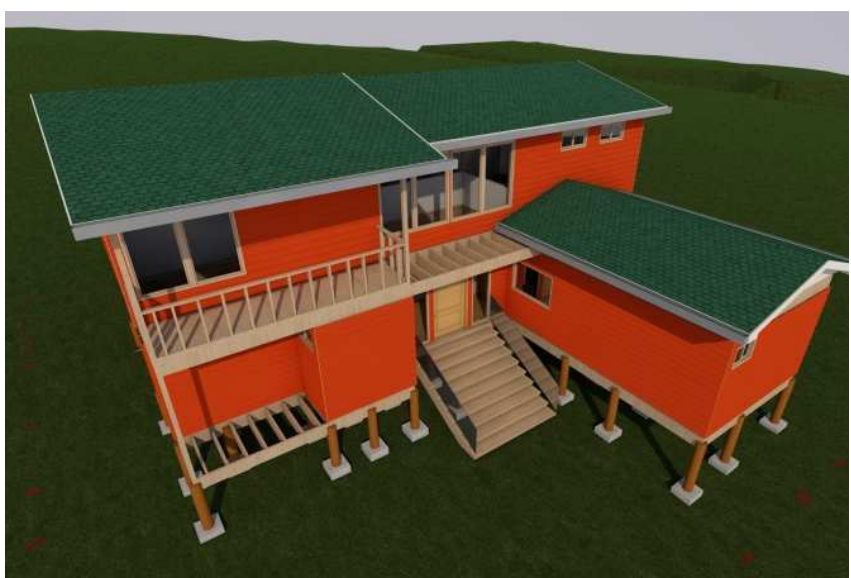

Figura 16: Revestimientos para un diseño de vivienda con Archiframe para Archicad. Fuente: Arq Victor Nuñez, 2017.

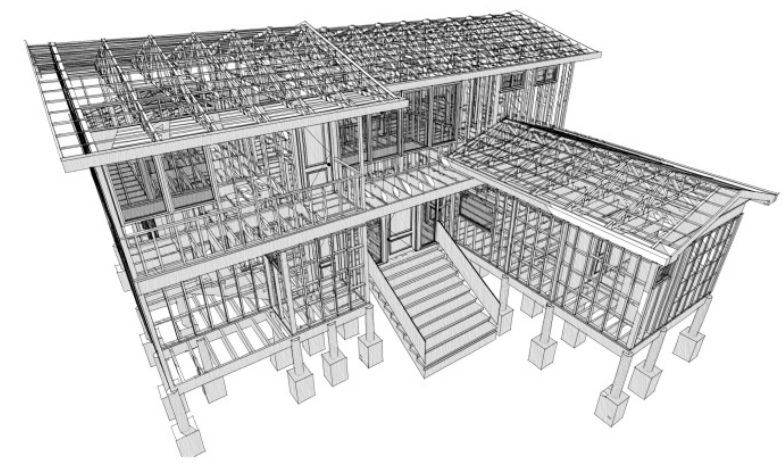

Figura 17: Entramados interiores para un diseño de vivienda con Archiframe para Archicad. Fuente: Arq Victor Nuñez, 2017.

La arquitecta Clara Codron exploró uno de los módulos de la extensión AGACAD Wood Framing (Wall+), mediante la plataforma REVIT. Acá fue posible configurar, diseñar y documentar todas las capas de los entramados verticales de un proyecto de vivienda en madera. En caso de cambio en el diseño de los entramados verticales de cualquier parámetro (distanciamiento, altura, etc.) el modelo se actualiza de manera automática.

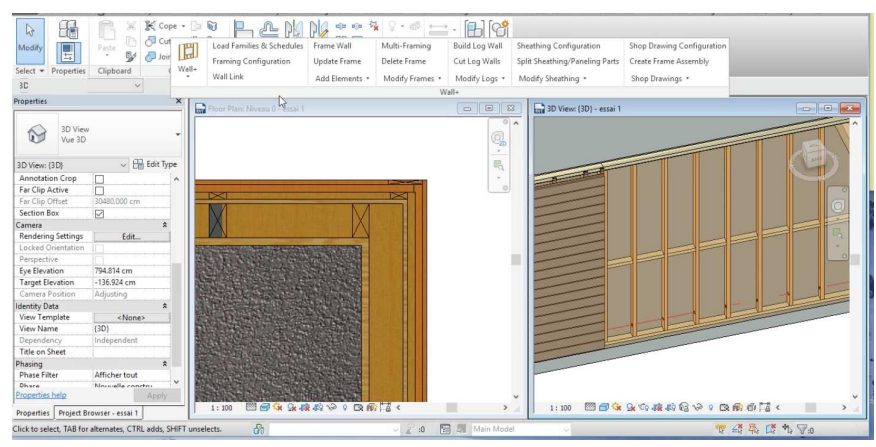

Figura 18: Vistas del modelo de entramados verticales hecho en AGACAD Wood Framing. Fuente: Clara Codron, 2017.

\section{CONCLUSIONES}

Actualmente, las herramientas BIM utilizadas para apoyar el diseño y construcción en madera (como Agacad y Archiframe) son escasas. Si bien permiten la creación de paquetes constructivos paramétricos, bibliotecas de materiales, rápida documentación y cubicación, ayuda a la fabricación, son complejos de usar, de pago y ninguno tiene soporte técnico en Chile. Otras como las provistas por Tekla y Autodesk, tienen aún muy poca documentación y casos de estudio conocidos, siendo necesario trabajo en aumentar su desarrollo y cobertura.

Los softwares BIM-madera serán necesarios para aumentar la productividad y sustentabilidad de la construcción en Chile, debiendo integrarse en iniciativas de apoyo a la madera que lideran centros tales como Maderas Universidad del BioBio, CIM-UC, Maderas USACH, CORMA, los cuales a la fecha no han integrado ni promovido el uso de estas plataformas.

En la actualidad, tanto en la academia, como en los planes gubernamentales en la materia, no hay una vinculación clara de la construcción en madera (diseño, construcción, operación) con las ventajas de los softwares BIM. Es recomendable que las diferentes agendas BIM y Madera trabajen de modo conjunto, mediante proyectos que integren ambas líneas en todos los frentes.

A continuación, una serie de recomendaciones y desafíos para una futura correcta implementación de BIM-Madera para Chile:

- Uso de software BIM y plugins en español. O mejorar en nivel de inglés de los actores (así además se mejora competitividad en mercados internacionales).

- Introducción de metodologías BIM y los Plugins en las mallas académicas de carreras AEC (Arquitectura, Ingeniería y Construcción).

- Creación de estándares nacionales de uso de BIM que considere el uso de madera.

- Crear cursos para profesionales del área AEC, tipo workshops, cursos o diplomados.

- Alianzas con productores de madera para construcción donde se discutan estandarizaciones de sus productos para plataformas BIM

- Seminarios de difusión apoyados por los gremios involucrados: Colegio de Arquitectos, Constructores e Ingenieros.

\section{AGRADECIMIENTOS}

Al grupo "BIM Madera" nacido inicialmente al alero del magister de Construcción Civil UC y acogido recientemente por la Universidad de las Américas, al director de Construcción Civil UDLA profesor Carlos Aguirre, al profesor UC Arq Andrés Sierra y a Construsoft Tekla (José Cosculluela). 


\section{Referencias}

AGACAD (2017) Retrieved from http://www.agacad.com/products/packages/wood-framing-solutions/(Accessed on: July, 2017)

ANTEL (2010) Diagnóstico de la situación actual de la coordinación proyectos en Chile, Santiago.

Archiframe (2017) Retrieved from http://www.archiframe.fi/en/inbrief/(Accessed on: July, 2017)

BSRIA (2015) Review of Global Environmental Assessment Methods Retrieved from https://www.bsria.co.uk/news/article/global-envassess/ (Accessed on: May 01, 2015)

CEI-BOIS (2009). Frente al cambio climático: Utiliza Madera. Digital Book by CEI-BOIS.org

CICRG (2010) Building Information Modeling Project Execution Planning Templates, The Computer Integrated Construction Research Group, The Pennsylvania State University.

CIM (2015) Centro UC de Innovación en Madera, Memoria Anual 2015, Santiago.

CONAF (2013) Por un Chile Forestal Sustentable, Ed. Baldini, A, CONAF Ediciones, Santiago: ZigZag.

Dzambazova, T; Krygiel, E and Demchak, G. (2008) Mastering Revit 2008, John Wiley and Sons, Indianapolis.

Eastman, C.; Teicholz, P.; Sacks, R.and Liston, K. (2011). BIM Handbook: A Guide to Building Information Modeling for Owners Managers, Designers, Engineers, and Contractors. 2nd ed., New Jersey, John Wiley and Sons,

Fleury, E. and Chiche, M. (2006). Rapport d'étude n DDD/DE/PEB 2006.096T: Le Bois dans la Construction, CSTB, France, p. 14.

GSA (2007) GSA BIM Guide Series 02. U.S. General Services Administration, Public Buildings Service, Office of the Chief Architect. Retrieved from www.gsa.gov/bim. Accessed on: June 01, 2013.

ISO 29481-1:2010(E): Building Information Modeling - Information Delivery Manual - Part 1: Methodology and Format

Khemlani, L. (2010), BIM Evaluation Study Report, AIA Large Firm Round Table (LFRT) CEO Committee.

Lobos, D. (2011). BIM Supported Building Envelopes and Space Layout Based on a Case Study in South America. Weimar, Bauhaus University Weimar Press, 211 p.

Loyola, M. (2016). Encuesta Nacional BIM 2016: Informe de Resultados. Santiago: Universidad de Chile. Disponible en: www.bim.uchilefau.cl

Madera 21 (2017) ¿Por qué Madera? Retrieved from http://www.madera21.cl/?page_id=2757 (Accessed on: July 2017)

McGraw-Hill (2012), The Business Value of BIM for Owners SmartMarket Report,

MINVU (2007) Ley General Urbanismo y Construcciones. DFL 458 y D.S. №103. Ministerio de la Vivienda del Gobierno de Chile, Santiago de Chile.

Organización de las Naciones Unidas para la Alimentación y la Agricultura (2015) Evaluación de los Recursos Forestales Mundiales, Roma.

Reddy, K.P. (2012) BIM For Building Owners And Developers Marking a Business case for using BIM on Projects, Wiley, New Jersey

Rojas, R (2011) Building Information Modeling - BIM. Red Interamericana de Centros de Innovación en la Construcción. White paper, 17 marzo de 2011.
Smith, P; (2005) Architecture in a climate of Change. A guide to sustainable design, Routledge; $2^{\text {nd }}$ Edition.

Soto, C; and Moa, C, (2013) Object Interaction Query: A Context Awareness Tool for Evaluating BIM Components' Interactions, SIGraDi 2013 [Proceedings of the 17th Conference of the Iberoamerican Society of Digital Graphics - ISBN: 978-956-705186-1] Chile - Valparaíso 20 - 22 November 2013, pp. 269 